\title{
Substituição de Materiais Poliméricos através de Seletores de Materiais e Fornecedores: Um Estudo de Caso na Indústria Automobilística
}

\author{
Isaias Bissoto, Thais H. Sydenstricker \\ Departamento de Engenharia Mecânica, UFPR
}

\begin{abstract}
Resumo: Neste trabalho foi avaliada a substituição de poliftalamida (PPA) da portinhola do tanque de combustível de um automóvel por outros polímeros. Foram utilizados e avaliados vários seletores de materiais disponíveis na Internet para a identificação de possíveis materiais substitutos à PPA. A PPA é um polímero de alto desempenho não produzido no Brasil, de custo elevado e de difícil processamento. Apesar de sua indicação de uso em peças da indústria automotiva devido às suas excelentes propriedades, sua substituição por outros plásticos implicaria em uma série de vantagens no processo produtivo. Após o resultado desta busca em sites, foram sugeridas melhorias para a elaboração de um novo seletor de materiais poliméricos. A avaliação final deste estudo de caso compara os resultados obtidos através da pesquisa nos seletores de materiais com aqueles obtidos através da consulta direta aos fornecedores locais de polímeros.
\end{abstract}

Palavras-chave: Seletor de materiais, poliftalamida, PPA, PC/PBT, indústria automotiva, logística.

\section{Substitution of Polyphthalamide (PPA) Used in the Fuel Tank Cap Lid of a Car by other Polymers}

Abstract: In this study, we evaluated the possible replacement of polyphthalamide (PPA) at the fuel tank cap lid of a car by other polymers. Various materials selectors available in the Internet were used to identify possible substitute materials to PPA. This material is a high-performance, high-cost, difficult-to-process polymer, which is not produced in Brazil. Despite its applicability to automotive parts due to its outstanding properties, its substitution by other polymers would imply in a series of advantages for the process of parts production. After the selectors' usage, a series of enhancements to these sites were suggested in order to make it possible to create a better layout of a materials selector for a future implementation. This study's final evaluation compares the final results obtained from the selectors with those from the local polymer suppliers.

Keywords: Materials selector, polyphthalamide, PPA, PC/PBT, automotive industry, logistics.

\section{Introdução}

A portinhola da tampa do tanque de combustível de um veículo montado na região metropolitana de Curitiba é produzida em poliftalamida, (PPA), via injeção de polímero em molde especificamente desenhado para o material. No entanto, o uso da PPA na peça de acabamento externo exige a aplicação de uma camada de primer antes de ser pintada, o polímero é importado, está associado a uma elevada complexidade logística para sua obtenção, apresenta alto custo, o processo de injeção é difícil e exige a utilização de um berço para a consolidação da forma final da peça. A peça fica sujeita a altos índices de perda por reprovação de qualidade e deficiências logísticas reduzem a produtividade do processo de produção.

A PPA foi escolhida como material para esta peça devido às suas propriedades superiores, mesmo nas condições mais adversas, como baixas temperaturas, comuns na Europa, onde o veículo foi projetado e também é produzido. A utili- zação de materiais mais comuns, como a poliamida reforçada com fibra de vidro não foi indicada inicialmente, pois, como o veículo é exportado para o Canadá onde temperaturas abaixo de $15{ }^{\circ} \mathrm{C}$ negativos são comuns, a peça se torna frágil. Além disso, como é um veículo que compete no segmento mais expressivo na Europa, o engenheiro construtor especificou um material que superasse as propriedades dos materiais empregados pelos concorrentes.

O presente trabalho visa analisar no estudo de caso apresentado, a busca por polímeros alternativos à PPA. A seleção de polímeros substitutos à PPA foi realizada nos principais seletores de materiais disponíveis na Internet ${ }^{[1-6]}$, permitindo a comparação e avaliação dos diferentes processos de busca e de seus resultados. Além da análise da adequação dos sites consultados ao estudo de caso, foram utilizados como parâmetros de crítica, algoritmos de seleção de materiais ${ }^{[7-10]}$. Foi realizada a comparação de resultados obtidos nos sites com os obtidos de fornecedores locais de polímeros. Finalmente, foram apresentadas sugestões para

Autor para Correspondência: Isaias Bissoto, Departamento de Engenharia Mecânica, UFPR, Caixa Postal 19011, CEP: 81531-990, Curitiba, PR. E-mail: isaiasbissoto@gmail.com 
o aumento da eficiência de busca em seletores de materiais plásticos $^{[12]}$.

\section{Experimental}

A busca por polímeros alternativos se fez de duas formas: i) consulta e uso de alguns sites da internet ${ }^{[1-6]}$ dedicados à seleção de materiais poliméricos e; ii) consulta direta a fornecedores tradicionais de materiais empregados na indústria automotiva, com grande experiência na seleção e desenvolvimento de materiais para os usos típicos. Com isto se pôde avaliar o desempenho destes seletores frente ao conhecimento obtido dos fabricantes de polímeros. A análise crítica decorrente desta comparação sustentou o estudo dos algoritmos computadorizados de seleção de materiais. Foram acessados os seguintes sites para a realização da busca ao material substituto:

- http://www.ticona.com/tools/search/lit_search.cfm ${ }^{[1]}$

- http://www.omnexus.com/tc/polymerselector/index.aspx ${ }^{[2]}$

- http://www.geplastics.com/dfss/servlet/matsel ${ }^{[3]}$

- http://www.matweb.com/search/SearchProperty.asp ${ }^{[4]}$

- http://www.designinsite.dk/[5]

- http://www.rhodia-ep.com/ep/ms_search_form.jsp ${ }^{[6]}$

Um relatório foi realizado, listando críticas e dificuldades enfrentadas na busca e dando sugestões para facilitar a seleção. Todas as alternativas dadas pelos sites foram listadas e avaliadas como substitutos à PPA.

Aos diversos fornecedores instalados no Brasil foi solicitado propor quais dos materiais em seus portfólios poderiam substituir a PPA, mesmo apresentando alguns desvios em relação à especificação proposta inicialmente pela companhia. Partiu-se não só das características físico-químicas dos polímeros, mas também da experiência anterior dos fornecedores no emprego de materiais em peças de acabamento externo de veículos.

Para a solução dos problemas de obtenção e disponibilidade da peça fez-se a caracterização dos materiais que eventualmente se enquadraram nos requisitos de projeto, bem como sua análise laboratorial e, se julgados compatíveis com o processo, executaram-se os testes de injeção conforme as orientações necessárias à obtenção da peça.

Quando julgado adequado, após os testes indicou-se ou não o emprego do material substituto.

\section{Resultados e Discussão}

\section{Processo de seleção de polímeros alternativos à PPA em sites de seletores de materiais}

Alguns dos sites de seletores de materiais utilizados são mais voltados à pesquisa científica e outros são mais voltados à venda de polímeros após contato com o cliente que fez a busca infrutífera. Não existe uma uniformização dos critérios de busca de materiais nestes seletores, pois cada um deles solicita os dados de entrada de acordo com seu perfil de finalidade, seja genérico ou comercial. Assim, o seletor
Matweb solicita informações técnicas como tensão de escoamento e resistência ao impacto, ao passo que os seletores "comerciais" se limitam a perguntar muitas vezes apenas o campo de aplicação.

O Quadro 1 apresenta as impressões e resultados obtidos da utilização destes sites para a busca de polímero alternativo à PPA no estudo de caso realizado.

Os resultados das buscas aos seletores não foram satisfatórios a este estudo de caso, pois as premissas básicas do desenvolvimento foram a manutenção da mesma ferramenta (molde de injeção) desenvolvida para a PPA com, no máximo, pequenas adaptações, e a manutenção da resistência ao impacto em baixas temperaturas da peça acabada. Ao considerar-se alguns dos polímeros sugeridos pelos seletores para o estudo, como foi o caso das poliamidas reforçadas com fibra de vidro, percebeu-se a necessidade de uma ferramenta nova ou alterações muito drásticas na ferramenta existente, além de o próprio polímero não resistir adequadamente aos testes de impacto a temperaturas baixas, típicas de países do hemisfério norte, inviabilizando sua aplicação. Os sites consultados não consideram a temperatura de aplicação da peça plástica e não prevêem a possibilidade de adaptação de um material a uma ferramenta já existente.

Os sites de fabricantes de polímeros são mais voltados às práticas de marketing e apoio às vendas, pois raramente detalham a busca. Sua maior vantagem é o de fazer um primeiro contato do cliente com a companhia, indicando dados superficiais que farão uma primeira seleção. Contudo, as peculiaridades de cada caso não são levadas em consideração, pois não se destinam a grandes refinamentos na busca.

\section{Avaliação dos seletores de materiais}

Após avaliar os sites objetos deste estudo, as conclusões seguintes e/ou sugestões de melhorias para torná-los mais amigáveis aos usuários incluem:

- Os sites de seletores devem apresentar um tutorial sobre o funcionamento do seletor ao usuário. Alguns sites solicitam valores de propriedades para o início da busca e seleção de possíveis candidatos. No entanto, em alguns casos o usuário não sabe informar a faixa de valores de determinada propriedade e pode induzir a busca a um erro, caso qualquer valor seja dado somente para tornar possível a continuação da busca. Portanto, os sites devem considerar a possibilidade da resposta "não sei" e continuar a busca através de outra estratégia. Alguns sites consideram a resposta "ignore", ou seja, consideram que a propriedade ou característica em questão é irrelevante ao usuário. O seletor deveria permitir aos usuários iniciar a busca conforme suas necessidades e seu conhecimento quanto ao projeto de produtos. Assim, o sistema de busca inicial deveria prever a possibilidade de seleção tanto via campo de aplicação (mercado, segmento, peça) quanto por propriedades físico-químico-mecânicas dos materiais.

- A ampliação da base de usuários pode ser executada através da escolha do sistema métrico (S.I. ou inglês) e da possibilidade de uso de três idiomas (português, espanhol e inglês).

- O layout do seletor deve ser organizado de forma a faci- 
Quadro 1. Comentários e resultados obtidos com a busca a sites de Seletores de Materiais

\begin{tabular}{|c|c|}
\hline Site * & Comentários e Resultados \\
\hline 1 & $\begin{array}{l}\text { Fonte de dados abrangente; } \\
\text { De uso aberto, acessível a qualquer usuário sem necessidade de cadastro prévio; } \\
\text { Inclui formas de processamento mais adequadas para cada polímero; } \\
\text { Informa faixas de custos para obtenção das peças; } \\
\text { Genérico para todos os tipos de materiais, com e sem marcas; } \\
\text { O software não indica material, apenas o grupo de materiais possíveis, neste caso, termoplásticos para injeção. }\end{array}$ \\
\hline 2 & $\begin{array}{l}\text { De uso aberto (não necessita cadastro prévio do usuário) para pesquisas básicas; requer cadastro para busca } \\
\text { avançada, disponibiliza a comparação entre materiais. Somente efetua buscas entre os produtos da companhia. } \\
\text { A busca se faz por propriedades e/ou campo de aplicação. Resultado da busca: a) para portinhola de combustível } \\
\text { de automóveis: POM } 30 \% \text { fibra de vidro, PPE 3-\% fibra de vidro, PPE com carga mineral ou PPA. B) para a } \\
\text { combinação das propriedades } 1,2 \text { e } 3 \text { do quadro 5: nenhum resultado obtido. }\end{array}$ \\
\hline 3 & $\begin{array}{l}\text { Precisa de cadastro prévio do usuário para inicialização e uso do seletor; } \\
\text { Apresenta tanto a ficha técnica dos polímeros quanto dados relativos à composição do material e categoria a que } \\
\text { pertence; } \\
\text { Busca pode ser feita pelas propriedades do material que se deseja; } \\
\text { Somente para produtos da companhia. Resultado da busca: PC + PBT, PC + Poliéster ou PC + ABS; } \\
\text { (com propriedades mecânicas especificadas, mas sem proporção dos materiais nas misturas) }\end{array}$ \\
\hline 4 & $\begin{array}{l}\text { De uso aberto (não necessita cadastro prévio do usuário) para pesquisas básicas; requer cadastro para busca } \\
\text { avançada, disponibiliza a comparação entre materiais e download de fichas técnicas; } \\
\text { Abrangente também, mas precisa de dados técnicos para fazer a seleção; } \\
\text { Necessário selecionar até três características técnicas para proceder à escolha na busca básica; } \\
\text { Estrutura de busca bastante técnica, apresentando nos resultados tanto a ficha técnica quanto dados da composição } \\
\text { do polímero, categoria a que pertence e processos de fabricação possíveis; } \\
\text { Retorna tanto materiais genéricos como produtos de marca; } \\
\text { Exige a abertura de várias telas, demanda longo tempo de busca e pode causar desorientação do usuário; } \\
\text { Resultados: nylon } 6 \text { ou } 66 \text { reforçados com fibra de vidro e/ou carga mineral ou } 10 \% \text { de fibra de carbono; } \\
\text { poliarilamida reforçada com fibra de vidro; PC reforçado com fibra de vidro; PBT reforçado com fibra de vidro; } \\
\text { PBT reforçado com fibra de vidro e PTFE; poliimida; PPS reforçado com } 40 \% \text { de fibra de vidro; poliacetal } \\
\text { copolímero reforçado com } 30 \% \text { de fibra de vidro; poliestireno sindiotático reforçado com fibra de vidro ou } \\
\text { carbono. }\end{array}$ \\
\hline 5 & $\begin{array}{l}\text { Busca apenas pela lista de aplicações já executadas pelo instituto, é apresentada uma lista com as características } \\
\text { do material proposto. A busca pelas propriedades do polímero não é considerada. Não apresenta valores das } \\
\text { propriedades do material proposto, apenas suas características. }\end{array}$ \\
\hline 6 & $\begin{array}{l}\text { Não requer cadastro prévio do usuário para uso; } \\
\text { Os dados de busca são os sugeridos pelo site, visando adequar ao portfólio de produtos da companhia. A } \\
\text { apresentação dos resultados é feita de forma comercial, mostrando sempre o nome comercial do produto da } \\
\text { companhia; É dada a ficha técnica completa, com muitas propriedades e valores, com possibilidade de consulta } \\
\text { direta à folha de dados do produto. } \\
\text { Resultados: poliamidas reforçadas da família TECNYL@. }\end{array}$ \\
\hline
\end{tabular}

* $1 \mathrm{http}: / /$ www.ticona.com/tools/search/lit_search.cfm

2 http://www.omnexus.com/tc/polymerselector/index.aspx

$3 \mathrm{http} / / / \mathrm{www} . g e p l a s t i c s . c o m / \mathrm{dfss} /$ servlet/matsel

4 http://www.matweb.com/search/SearchProperty.asp

5 http://www.designinsite.dk/

6 http://www.rhodia-ep.com/ep/ms_search_form.jsp

litar visualmente o acesso (ser amigável ao usuário). Vários sites consultados são visualmente poluídos.

- Deve existir um compromisso entre a usabilidade e a correção dos resultados obtidos. Inúmeros questionamentos relevantes a cada aplicação particular não são contemplados normalmente, como os relativos a filmes multicamadas, o tempo de serviço do produto em questão, questões relativas ao impacto ao meio ambiente causado pelo uso daquele polímero, entre outros. Seria necessário, a partir do produto que se deseja, dirigir o seletor a outras máscaras específicas, que considerassem as particularidades de cada aplicação, de forma que se possa permanecer em um número de questões em torno de 20 até uma primeira seleção, com tantas iterações posteriores para refinar a busca quantas sejam necessárias e o usuário esteja disposto a executar.

- Há várias formas de se proceder a pesquisa básica em seletores de materiais. O critério mais comum usado em seletores voltados à pesquisa é o de propriedades mecânicas, físicas e químicas. A principal limitação deste método é a desconsideração quanto às características finais de uso, pois 
os dados empregados e solicitados para a seleção são os de polímero recém produzido, não levando em conta as características do ambiente a que o produto será exposto durante o uso. Além disto, o usuário pode não saber responder a um número suficiente de questões sobre propriedades físicas, necessárias para uma correta indicação de material. Assim, questões ao usuário sobre características do material buscado, sem listagem exaustiva de valores de propriedades específicas poderiam ser formuladas, por exemplo, se o software tentasse localizar propriedades térmicas importantes como temperatura de transição vítrea $\left(\mathrm{T}_{\mathrm{g}}\right)$ e temperatura de fusão cristalina $\left(T_{m}\right)$ usando equações disponíveis na literatura ${ }^{[11]} \mathrm{e}$ dados como módulo de cisalhamento e aspecto do polímero para a aplicação buscada (ex, rígido ou flexível).

- Um seletor de materiais que contemple esta e outras particularidades pode ser viabilizado comercialmente, desde que haja um esforço conjunto neste sentido. Se houvesse um acordo com os vários fornecedores, que fabricam e importam polímeros e que possuem os dados técnicos para a divulgação, visando construir um banco de dados nacional abrangente, um seletor de materiais eficiente e amigável ao usuário poderia ser construído.

A outra forma de pesquisa básica encontrada foi a empregada por um instituto de design dinamarquês ${ }^{[5]}$ para os materiais já empregados nos produtos por ele desenvolvidos. Indiscutivelmente esta é uma forma de busca razoavelmente eficiente para um usuário com elevado conhecimento na especificação de materiais poliméricos no projeto de produtos e consiste em uma ferramenta útil para aquele projetista com apenas uma idéia de partida, sem saber exatamente que material ou grupo de materiais poderia empregar. Deve, no entanto ser usada com muito cuidado, pois pode induzir a erros grosseiros, uma vez que, novamente, não se fala em tempo de uso ou ambiente de exposição do produto final.

\section{Orientações para construção de um site para seletor de materiais poliméricos}

Foram feitas propostas para o projeto de um novo seletor de materiais plásticos que devem ser interpretadas como um

Quadro 2. Encadeamento proposto para o banco de dados do seletor. roteiro para a construção de um novo site para uma busca eficiente de materiais poliméricos.

Com o objetivo de apresentar uma nova idéia às diferentes formas de busca para materiais poliméricos dentro dos sites de seleção de materiais, visando aperfeiçoar o processo de busca tanto para usuários "leigos" como para auxiliar os projetistas e designers ambientados com o processo de seleção, propõem-se os seguintes passos e forma de seleção:

O código do seletor (Quadro 2) deve ter um detalhamento de programação tal que se construa um banco de dados que relacione categorias de produtos com tipos de produtos com estruturas possíveis, com geometrias adequadas, com dimensões do produto e com o âmbito da aplicação. O nível de detalhamento exemplificado no Quadro 2 para a categoria "automotivo" deve ser reproduzido para as outras, englobando as peculiaridades de cada uma. Demonstra-se aqui apenas um exemplo em cada nível visando à compreensão da idéia. Em todas as etapas deve ser permitido ao usuário inserir a resposta "não sei" ou "ignore" (dado irrelevante) previamente informado do significado destas expressões.

Seguindo-se a lógica concebida para a busca, neste nível o usuário escolheria seqüencialmente a categoria, o tipo e assim sucessivamente até o nível mais baixo da cadeia de busca. Assim apresentar-se-ia o critério de busca por categoria do produto, listando-se materiais específicos para aquele grupo indicado. Ao avançar a busca aos níveis mais baixos, haveria um estreitamento dos materiais disponíveis até o nível de escolha do usuário. Ao aprofundar-se, o sistema faria as correlações devidas entre estrutura, geometria e dimensões do produto para definir qual o melhor método de produção (roto moldagem, termo formação, injeção, extrusão etc.) da peça desejada e sugerí-lo ao usuário quando da apresentação dos resultados. Opcionalmente poderá o usuário indicar o processo produtivo que julgue apropriado, em função da disponibilidade do equipamento, por exemplo.

Caso o usuário prefira, pode ser alternativamente executada a inserção das propriedades físico-químicas ou mecânicas dos polímeros pretendidos. Neste modo de busca, o seletor se guia pelos limites máximos e/ou mínimos das propriedades individuais informadas em comparação com os dados

\begin{tabular}{c|c|c|c|c|c}
\hline Categoria & Tipo & Estrutura & Geometria & Dimensões & Contexto \\
\hline Automotivo & Pára-choque & \multirow{2}{*}{ Sem reforço } & Plana & Área $<0.5 \mathrm{~m}^{2}$ & Acabamento externo \\
\cline { 4 - 6 } & & & & & Exposição UV \\
\cline { 4 - 6 } & & & & Choque físico \\
\cline { 4 - 6 } & & & Espacial & Volume $<0.5 \mathrm{~m}^{3}$ & \\
\cline { 4 - 6 } & & & Complexa & & \\
\cline { 4 - 6 } & & & & & \\
\hline Eletro-doméstico & & & & & \\
\hline Móvel & & & & & \\
\hline
\end{tabular}


Quadro 3. Interface homem-máquina proposta para o modelo de seletor de materiais.

\begin{tabular}{l|l|l|l}
\hline $\begin{array}{c}\text { Comandos } \\
\text { do usuário }\end{array}$ & \multicolumn{1}{|c|}{ Ação esperada do usuário } & \multicolumn{1}{c}{ Resposta do sistema } & Ação esperada do sistema \\
\hline $\begin{array}{l}\text { Inserção dos } \\
\text { dados básicos }\end{array}$ & $\begin{array}{l}\text { Seleção do tipo de produto na lista } \\
\text { do software ou Inclusão dos dados } \\
\text { básicos ou } \\
\text { Inclusão da forma geométrica } \\
\text { aproximada do produto }\end{array}$ & Consulta aos bancos de dados & $\begin{array}{l}\text { Associar o pedido do usuário ao(s) } \\
\text { material(is) correlato(s) }\end{array}$ \\
\hline $\begin{array}{l}\text { Detalhar os } \\
\text { requisitos }\end{array}$ & Refinar os dados inseridos & $\begin{array}{l}\text { Separar os materiais que atendem } \\
\text { aos critérios }\end{array}$ & $\begin{array}{l}\text { Formar um banco de dados com os } \\
\text { resultados da pesquisa }\end{array}$ \\
\hline
\end{tabular}

armazenados nos bancos de dados de materiais do seletor, a exemplo dos critérios adotados pelo Matweb.

Visando a uma busca simplificada, o seletor indicaria ao usuário, a exemplo do Designinsite, a lista de materiais conhecidos já empregados na fabricação de um determinado produto. Assim, caso o usuário deseje projetar uma cadeira em material polimérico, o site deve mostrar-lhe os diversos tipos de cadeiras disponíveis em seu banco de dados já conhecidas, listando os materiais empregados para o fim específico. Caberá ao usuário concordar ou não com a indicação ou, alternativamente, reiniciar o processo de busca utilizando-se de um dos outros critérios adotados pelo seletor.

Com a indicação dos dados iniciais pelo usuário, o sistema deve consultar os bancos de dados, correlacionando os dados inseridos com aqueles armazenados em seus bancos de dados para selecionar os materiais prováveis, formando uma primeira listagem de polímeros candidatos à aplicação buscada. Num nível seguinte, o usuário é solicitado a refinar a busca, indicando condições específicas ao seu caso, como temperatura de operação, prazo de vida útil desejado, transparência, necessidade de baixa permeabilidade a gases, entre outras características peculiares. Uma nova seleção e a indicação ao usuário dos novos resultados viriam a seguir.

Estabelecendo-se o vínculo (Quadro 3) entre os comandos que devem ser inseridos pelo usuário com as ações esperadas as respostas do sistema associadas às ações deste, chega-se à seguinte interface homem-máquina:

O quadro deve ser interpretado da esquerda para a direita e de cima para baixo, associando-se a cada ação do usuário a respectiva resposta do sistema listada no mesmo nível.

Num segundo nível de detalhamento da proposta (Quadro 4), apresenta-se o encadeamento dos comandos do usuário necessários e as ações executadas pelo software para conclusão da tarefa proposta.

Com o intuito de simplificar a análise de resultados a apresentação dos resultados o sistema deveria considerar, além da listagem como os seletores atualmente apresentam, a construção de um gráfico "teia de aranha" como o da Figura 1, mostrando como as propriedades requeridas são atendidas dentro da escala proposta. Esse procedimento permite ao usuá- rio avaliar se o não atendimento por inteiro da variável específica compromete a aplicação ou se esta pode ser analisada com mais critério num teste experimental. Permite também a comparação de um polímero ideal hipotético com os disponíveis e o material a ser substituído.

O ideal seria poder contar com as três técnicas interligadas, utilizando-se o critério de materiais indicados para um segmento/produto para uma primeira seleção, em seguida o critério de propriedades mecânicas e físico-químicas, para, finalmente, oferecer ao usuário a ficha técnica dos materiais selecionados e o auxílio de um técnico, no caso de necessidade de esclarecimento de dúvidas ou de consulta a situações particulares não previstas no escopo do seletor.

Na hipótese de novas situações imprevistas anteriormente, o site deveria ser, depois de comprovada a falta de cobertura, atualizado para prever a nova condição, mantendo-se constantemente atualizado com o último nível de informação técnica disponível.

Um convênio celebrado entre os responsáveis pelo seletor idealizado e os diversos fabricantes ou comerciantes de polímeros (no caso de importados) pode ser a fonte do banco de dados dos polímeros a serem considerados na seleção, oferecendo sempre ao usuário grades comercialmente disponíveis em vez de simples indicação de propriedades teóricas, obtidas de manuais e catálogos sem conexão com os polímeros disponíveis para uso industrial. A atualização dos dados dos polímeros mantidos no banco de dados consolidado seria de encargo dos responsáveis pela comercialização, com direito a merchandising como forma de compensação pela participação e correção dos dados fornecidos. Oferecer o site como forma de contato de clientes com as companhias pode ser outra forma de viabilização do projeto.

Após a avaliação de diferentes polímeros sugeridos por fornecedores da montadora (PPA de outra fonte e poliamidas reforçadas), optou-se pelo uso da mistura PC/PBT 20/80. Analisada a mistura PC/PBT conforme a norma da montadora para materiais de acabamento externo (Quadro 5), verificouse que o material proposto atende às solicitações requeridas, apresentando boa estabilidade dimensional, atendendo aos requisitos de pintura superficial, sendo o material finalmen- 
Quadro 4 - Detalhamento do funcionamento do seletor proposto.

\begin{tabular}{|c|c|c|c|}
\hline Nível da aplicação & Comandos do usuário & Resposta do sistema & Resultados obtidos \\
\hline \multirow{6}{*}{ Inserir } & Indicar a categoria do produto & $\begin{array}{l}\text { Restringe no banco de dados só os } \\
\text { materiais destinados à categoria } \\
\text { pretendida }\end{array}$ & \multirow{6}{*}{$\begin{array}{l}\text { Forma-se o primeiro grupo de } \\
\text { materiais possíveis de serem } \\
\text { aplicados ao caso concreto. } \\
\text { Pode a cada etapa mostrar ao } \\
\text { usuário quantos materiais foram } \\
\text { desconsiderados com o novo } \\
\text { critério e quantos ainda restam. } \\
\text { Armazena os critérios para } \\
\text { alteração ou consulta ou novos } \\
\text { cálculos. }\end{array}$} \\
\hline & Indicar o tipo de produto & $\begin{array}{l}\text { Restringe no resultado anterior só } \\
\text { os materiais aplicáveis ao tipo } \\
\text { pretendido }\end{array}$ & \\
\hline & Indicar a estrutura pretendida & $\begin{array}{l}\text { Restringe novamente o resultado } \\
\text { anterior àqueles materiais que } \\
\text { atendam ao critério estrutural. }\end{array}$ & \\
\hline & Indicar a geometria & $\begin{array}{l}\text { Nova restrição, permanecendo só } \\
\text { os materiais adequados à geome- } \\
\text { tria proposta. }\end{array}$ & \\
\hline & Indicar as dimensões & $\begin{array}{l}\text { Verifica eventuais restrições aos } \\
\text { materiais restantes. }\end{array}$ & \\
\hline & Indicar o âmbito de aplicação & $\begin{array}{l}\text { Exclui do resultado materiais que } \\
\text { não atendam às exigências im- } \\
\text { postas. }\end{array}$ & \\
\hline Detalhar & $\begin{array}{l}\text { Selecionar características adicio- } \\
\text { nais ao projeto (Uso de material } \\
\text { reciclado, Filme multicamada, } \\
\text { reciclabilidade) }\end{array}$ & $\begin{array}{l}\text { Verifica nos resultados quais não } \\
\text { atendem aos novos requisitos e } \\
\text { exclui do resultado. }\end{array}$ & $\begin{array}{l}\text { Escolha no resultado anterior } \\
\text { daqueles com maior probabi- } \\
\text { lidade para atender aos requi- } \\
\text { sitos solicitados }\end{array}$ \\
\hline \multirow{3}{*}{ Concluir } & $\begin{array}{l}\text { Indicação do processo de fabri- } \\
\text { cação (Opcional) }\end{array}$ & $\begin{array}{l}\text { Indicação do processo de fabri- } \\
\text { cação mais adequado caso o } \\
\text { usuário não restrinja }\end{array}$ & \multirow{3}{*}{$\begin{array}{l}\text { Indicação dos materiais mais } \\
\text { prováveis para atender às solici- } \\
\text { tações }\end{array}$} \\
\hline & Restrição ao custo do material & $\begin{array}{l}\text { Verifica nos resultados quais não } \\
\text { atendem aos novos requisitos e } \\
\text { exclui do resultado }\end{array}$ & \\
\hline & Outros critérios & & \\
\hline
\end{tabular}

Quadro 5. Ensaios realizados e resultados obtidos: PC/PBT x PPA x Especificação mínima.

\begin{tabular}{|c|c|c|c|c|}
\hline Nr. & Ensaio & PPA & PC-PBT & Mínimo \\
\hline 1 & Densidade DIN $53479, \mathrm{~g} / \mathrm{cm}^{3}$ & 1,53 & 1,2 & 1,1 \\
\hline 2 & Tensão de escoamento EN ISO 527-2 (velocidade $100 \mathrm{~mm} / \mathrm{min}$ ), N/mm² & 107 & 57,1 & 50 \\
\hline 3 & Alongamento até ruptura EN ISO 527-2 (velocidade $100 \mathrm{~mm} / \mathrm{min}$ ), $\%$ & 60 & 47,4 & 40 \\
\hline 4 & Resist. Deformação térmica VICAT, processo B50 EN ISO $306,{ }^{\circ} \mathrm{C}$ & 110 & 127,5 & 110 \\
\hline 5 & Dureza compressão esfera DIN EN ISO 2039-1 (358 N/30 seg), N/mm² & 125 & 98,2 & 95 \\
\hline 6 & Resist. Impacto sem entalhe, $23{ }^{\circ} \mathrm{C}$ EN ISO $179-1, \mathrm{KJ} / \mathrm{m}^{2}$ & 200 & 100 & 90 \\
\hline 7 & Resist. Impacto com entalhe, $23{ }^{\circ} \mathrm{C}$ EN ISO $179-1, \mathrm{KJ} / \mathrm{m}^{2}$ & 48 & 39,2 & 35 \\
\hline 8 & Resist. tração EN ISO 527-2 (velocidade $100 \mathrm{~mm} / \mathrm{min}$ ), N/mm² & 96 & 43,4 & 40 \\
\hline
\end{tabular}

te homologado pela montadora para o fim específico. No Quadro 5 apresentam-se os dados analíticos da mistura PC/ PBT, da PPA e a especificação mínima aceita pela montadora para materiais desta peça. Assim, o seletor de materiais pro- posto neste trabalho usará o estudo de caso discutido para a apresentação de resultados de uma busca hipotética. Na Figura 1, cada um dos raios da teia representa uma das propriedades enumeradas no Quadro 5. 


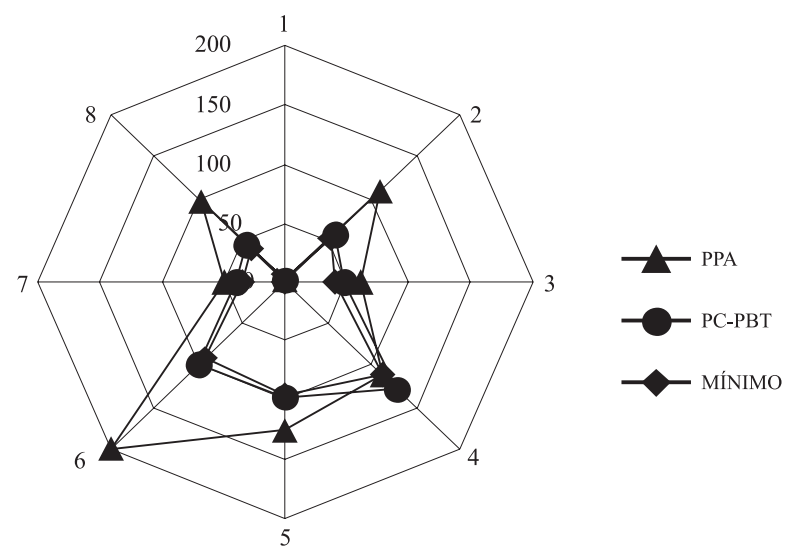

Figura 1. Análise comparativa: PC/PBT x especificação PPA

\section{Conclusões}

A busca por polímeros substitutos à PPA em sites da Internet no estudo de caso realizado não foi eficiente, pois não foi encontrado nenhum seletor de materiais que fizesse uma seleção criteriosa, justificando gastos da montadora em testes preliminares de injeção e mecânicos da peça em estudo.

O estudo quanto aos seletores de materiais comerciais atualmente disponíveis no mercado mostra que eles poderiam beneficiar-se das conclusões obtidas neste estudo exploratório. A incorporação de pequenas melhorias nestes programas de computador e a utilização de bancos de dados mais abrangentes dos fornecedores de polímeros no Brasil geraria usuários mais satisfeitos, com resultados de busca mais condizentes com os interesses dos usuários, trazendo substancial economia no processo de seleção de materiais plásticos.

A construção de um seletor de materiais que pudesse receber as informações dos materiais disponíveis dos fabricantes/comerciantes de polímeros no Brasil, de fonte nacional ou importada, com base no estudo e nas orientações apresentadas nos itens 3.2 e 3.3. traria grande benefício não só aos usuários, como também à comunidade científica envolvida com o projeto de produtos com matérias-primas poliméricas.

Mesmo o site Omnexus [2], que incorpora algumas das sugestões aqui apresentadas, não aponta o polímero resultado da indicação da experiência de fabricantes e técnicos especializados.

A substituição da PPA pela mistura PC/PBT 20/80, material de menor custo, traria vantagens ao processo de produção da peça do estudo de caso realizado e ao gerenciamento da produção e da cadeia logística de suprimento, tanto do fabricante da portinhola de combustível quanto para a montadora de automóveis.

\section{Referências Bibliográficas}

1. Seletor de Materiais. Disponível em <http://www.ticona.com/ tools/search/lit_search.cfm> Acesso em jul 2003 e set 2004.

2. Seletor de Materiais. Disponível em $<\mathrm{http}$ ://www.omnexus. com/tc/polymerselector/index.aspx $>$ Acesso em Abr/06.

3. Seletor de Materiais. Disponível em $<$ http://www. geplastics.com> Acesso em Jul/03.

4. Seletor de Materiais. Disponível em $<$ http://www. matweb.com/search/SearchProperty.asp $>$ Acesso em $\mathrm{Jul} / 03$ e Set/04.

5. Seletor de Materiais. Disponível em <http://www. designinsite.dk/> Acesso em jul 2003 e set 2004.

6. Seletor de Materiais. Disponível em $<$ http://www.rhodiaep.com/ep/ms_search_form.jsp> Acesso em jul 2003 e set 2004 .

7. Shercliff, H.R.; Lovatt, A.M. - Progr. in Mat. Sci., 46, p. 429-459 (2001).

8. Trethewey, K. R.; Wood, R. J. K.; Puget, Y. \& Roberge, P. R. - Mat. and Design, 19, p. 39-56 (1998).

9. Waterman, N. A. \& Ashby, M. F. (Ed.). "The Materials Selector", 2. ed., Chapman and Hall, London (1997).

10. Shanian, A. \& Savadogo, O. - Mat. and Design (in Press) (2004).

11. Van Krevelen, D.W. - "Properties of Polymers - their correlation with chemical structures, their numerical estimation and prediction from additive group contributions", Elsevier, Amsterdam (1990).

12. Bissoto, I.; "Substituição de materiais poliméricos através de seletores de materiais e fornecedores: um estudo de caso"; Dissertação (Mestrado); Setor de Tecnologia, UFPR. Curitiba, (2005).

Enviado: 12/09/05

Reenviado: 18/05/06

Aprovado: 20/05/06 